\title{
Genetic Influences on Sociability: Heightened Amygdala Reactivity and Event-Related Responses to Positive Social Stimuli in Williams Syndrome
}

\author{
Brian W. Haas, ${ }^{1 \star}$ Debra Mills, ${ }^{2 \star}$ Anna Yam, ${ }^{3}$ Fumiko Hoeft, ${ }^{1}$ Ursula Bellugi, ${ }^{3}$ and Allan Reiss ${ }^{1}$ \\ ${ }^{1}$ Center of Interdisciplinary Brain Sciences Research, Stanford University School of Medicine, Palo Alto, California 94305-5795, 25chool of Psychology, \\ Bangor University, Gwynedd LL57 2AS, United Kingdom, and ${ }^{3}$ Laboratory for Cognitive Neuroscience, Salk Institute for Biological Studies, La Jolla, \\ California 92037
}

\begin{abstract}
Williams syndrome (WS) is a genetic disorder caused by a hemizygous microdeletion on chromosome 7q11.23. WS is associated with a compelling neurocognitive profile characterized by relative deficits in visuospatial function, relative strengths in face and language processing, and enhanced drive toward social engagement. We used a combined functional magnetic resonance imaging (fMRI) and event-related potential (ERP) approach to examine the neural basis of social responsiveness in WS participants to two types of social stimuli, negative (fearful) and positive (happy) emotional facial expressions. Here, we report a double dissociation consistent across both methods such that WS participants exhibited heightened amygdala reactivity to positive (happy) social stimuli and absent or attenuated amygdala reactivity to negative (fearful) social stimuli, compared with controls. The fMRI findings indicate that atypical social processing in WS may be rooted in altered development of disparate amygdalar nuclei that subserve different social functions. The ERP findings suggest that abnormal amygdala reactivity in WS may possibly function to increase attention to and encoding of happy expressions and to decrease arousal to fearful expressions. This study provides the first evidence that the genetic deletion associated with WS influences the function of the amygdala to be particularly responsive to socially appetitive stimuli.
\end{abstract}

Key words: Williams syndrome; genetics; fMRI; emotions; amygdala; ERP

\section{Introduction}

Studying the neural basis of social cognition in individuals with well defined genetic etiologies advances our understanding of how genes influence social behavior. Williams syndrome (WS) is a genetic condition caused by a hemizygous microdeletion on chromosome 7q11.23. Individuals with WS are characterized by a distinctive neuropsychological profile comprised of deficient visuospatial function (Meyer-Lindenberg et al., 2004), relative strengths in language (Mervis and Becerra, 2007) and face (Mobbs et al., 2004; Santos et al., 2008) processing, and a tendency for enhanced affiliative drive (Jones et al., 2000; Doyle et al., 2004). For example, those with WS are less socially inhibited (Doyle et al., 2004), more likely to rate emotional facial expressions as approachable (Frigerio et al., 2006), and more gregarious (Klein-Tasman and Mervis, 2003) than mentally aged control groups. Although enhanced drive for social interaction in Williams syndrome has been reported using several experimental (Bellugi et al., 1999; Jones et al., 2000; Mervis and Klein-Tasman,

\footnotetext{
Received Nov. 5, 2008; revised Dec. 5, 2008; accepted Dec. 16, 2008.

This work was supported by National Institute of Child Health and Human Development Grant P01 HD033113-12 and National Institute of Mental Health Grant T32 MH19908.

*B.W.H. and D.M. contributed equally to this work.

Correspondence should be addressed to Allan L. Reiss, Department of Psychiatry and Behavioral Sciences, Stanford University School of Medicine, 401 Quarry Road, Stanford, CA 94305-5719. E-mail: areiss1@stanford.edu. D0I:10.1523/JNEUROSC1.5324-08.2009

Copyright $\odot 2009$ Society for Neuroscience $\quad 0270-6474 / 09 / 291132-08 \$ 15.00 / 0$
}

2000; Klein-Tasman and Mervis, 2003; Deutsch et al., 2007) and cross-cultural (Zitzer-Comfort et al., 2007) approaches, the neural basis of social cognitive function in WS remains poorly understood.

One brain region known to be involved in social functions such as affiliative processes is the amygdala (Skuse et al., 2003). The amygdala codes for the social/emotional salience of both negatively and positively valenced information (Costafreda et al., 2008; Paton et al., 2006), and damage to this structure results in profound abnormalities in social functioning (Aggleton, 2000). Indeed, previous studies have reported functional abnormalities of the amygdala in WS. For example, compared with typically developing controls, WS individuals exhibit diminished amygdala response to fearful stimuli (Meyer-Lindenberg et al., 2005) and heightened amygdala response to music (Levitin et al., 2003). It is unknown, however, if individuals with WS exhibit functional abnormalities when processing positively valenced social/emotional stimuli such as happy facial expressions. Is this study, we tested the hypothesis that those with WS exhibit heightened amygdala response to socially appetitive (happy) stimuli and diminished amygdala response to negative (fearful) social stimuli. We chose to measure amygdala function by means of two complementary methods, functional magnetic resonance imaging (fMRI) and event-related potentials (ERPs), using identical stimuli and a similar experimental design. fMRI provides the opportunity to spatially localize differences in activation during each 
condition, while ERP provides the opportunity to more accurately dissociate temporal components during each condition. Based on reports that those with WS are relatively hypersocial (Doyle et al., 2004) and have a particular affinity toward happy facial expressions (compared with mentally aged controls) (Frigerio et al., 2006), we predicted that we would observe heightened amygdala response during the processing of happy facial expressions in WS compared with controls. Additionally, based on findings demonstrating that those with WS exhibit reduced amygdala response to fearful social stimuli (Meyer-Lindenberg et al., 2005), we predicted that we would observe a similar pattern as assessed by both fMRI and ERP.

\section{Materials and Methods}

Participants. Twenty-seven subjects [13 typically developing (TD), 5 males; and 14 WS, 7 males] participated in the fMRI study, and seventy subjects [ 25 TD, 11 males; $30 \mathrm{WS}, 11$ males; and 15, 8 males with developmental delay (DD) of unspecified etiology] participated in the ERP study. Eleven subjects with WS [ 4 males, mean $(\mathrm{M})=31.13, \mathrm{SD}=7.55$ ] participated in both fMRI scanning and ERP recording. Subjects were excluded from participating if they had a history of psychiatric or neurologic problems as assessed by the Symptom Checklist-90-R (SCL90-R) (Derogatis, 1977). All included subjects had SCL-90-R scores that fell within one SD of a normative sample. Subjects were also excluded if they reported any current use of mood-altering medication, substance abuse during the 6 months before scan or any standard MRI contraindications.

WS participants were recruited as part of an ongoing multicenter collaborative research study focused on investigating the functional neuroanatomy of WS ( 33 total; 13 males; mean age $=31.01$; $\mathrm{SD}=8.80$ ). The diagnosis of WS was genetically confirmed in all participants using the fluorescent in situ hybridization test for a deletion of one copy of the elastin gene on chromosome 7. TD subjects were recruited locally (Palo Alto, CA for fMRI and San Diego, CA for ERPs) and were financially compensated for their participation [ 38 total; 18 males; $\mathrm{M}=29.71$; $\mathrm{SD}=$ 9.52; intelligence quotient (IQ): $\mathrm{M}=106, \mathrm{SD}=11.8$ ]. DD participants were recruited locally (San Diego, CA) through community media and state-run agencies (total $15 ; 8$ males; $\mathrm{M}=28.07, \mathrm{SD}=8.8$; IQ: $\mathrm{M}=62$, $\mathrm{SD}=8.8$ ). Criteria for participation in the DD group included developmental delay of unknown etiology without the presence of symptoms indicative of an autism spectrum disorder. This study was approved by the Stanford University and Salk Institute Administrative Panel on $\mathrm{Hu}-$ man Subjects in Medical Research

There were no significant differences in age $\left(F_{(2,67)}=0.600, p=0.552\right)$ or proportion of males to females between the groups $\left(\chi_{(2)}^{2}=1.155, N=\right.$ $70, p=0.561)$. The WS and DD groups were matched on full-scale IQ (WS: $\mathrm{M}=65, \mathrm{SD}=6.8$; $\mathrm{DD}: \mathrm{M}=62, \mathrm{SD}=8.8 ; t_{(43)}=1.148, p=0.153$ ).

Task design. The stimuli consisted of color pictures of headshots of young adults displaying happy, fearful, and neutral expressions. One hundred undergraduate students were trained to display emotional expressions depicting a variety of emotional expressions including happy, fearful, and neutral. Each photograph was rated by 20 students on a five-point Likert scale for how typical each photograph depicted each emotional category with 1 scored as "not at all like the emotion" and 5 scored as "very characteristic of the emotion." Only stimuli that had the highest average ratings for a given target emotion were selected for that category. Fearful face stimuli were rated as more fearful than neutral $(t=$ $16.01, p<0.001)$ and happy $(t=18.65, p<0.001)$ faces, neutral face stimuli were rated as more neutral than fearful $(t=36.63, p<0.001)$ and happy $(t=47.54, p=0.001)$ faces, and happy face stimuli were rated as more happy than fearful $(t=61.93, p<0.001)$ and neutral $(t=49.66$, $p<0.001$ ) faces. A group of randomly selected photographs were selected to create scrambled isoluminant images that were divided into 256 parts as in a previous study (Mobbs et al., 2004).

Both the fMRI and ERP tasks were event-related designs with four experimental conditions (happy, fearful, neutral, and scrambled) and a resting baseline. Subjects were instructed to judge if each face was male, female, or scrambled by responding with their right index, middle, or ring finger as quickly and as accurately as possible. In the fMRI task, there were a total of 30 trials per condition. Each stimulus was presented for $1750 \mathrm{~ms}$, followed by a $250 \mathrm{~ms}$ duration fixation cross. There were two runs, with each run lasting $4 \mathrm{~min} 32 \mathrm{~s}$. In the ERP task, there were a total of 50 trials per condition. Each stimulus was presented for $1750 \mathrm{~ms}$, followed by a $250 \mathrm{~ms}$ duration fixation cross. The intertrial interval was random with a mean of $1000 \mathrm{~ms}$ (range $=800-1200 \mathrm{~ms}$ ). The random order of the intertrial interval was determined by using Presentation software (Neurobehavioral Systems). Behavioral responses were collected within the $2000 \mathrm{~ms}$ following the onset of each stimulus in both the fMRI and ERP task.

$f M R I$ data acquisition. Whole-brain imaging data were acquired on a GE-Signa 3 T scanner (General Electric). For structural whole-brain images, a three-dimensional high-resolution spoiled gradient scan (SPGR) (repetition time, $24 \mathrm{~ms}$; echo time, $5 \mathrm{~ms}$; flip angle, $15^{\circ}$; number of excitations, 2 ; matrix size, $256 \times 256$; field of view, $24 \mathrm{~cm}$; slice thickness, $1.2 \mathrm{~mm} ; 124$ contiguous slices) and a T1 in-plane scan (14 slices, $5 \mathrm{~mm}$ thickness; oriented parallel to the line between the anterior and posterior commissure) were conducted. Functional images were acquired using a gradient echo $\mathrm{T} 2{ }^{*}$-weighted echoplanar imaging scan and were obtained using a flip angle of $80^{\circ}$, repetition time $=2.0 \mathrm{~s}$, echo time $=30 \mathrm{~ms}, 32$ slices, and a field of view $=200 \times 200 \mathrm{~mm}$ matrix.

Functional data were preprocessed and statistically analyzed using SPM2 (Wellcome Department of Imaging Neuroscience, London, UK). The images were temporally realigned to the middle slice and spatially realigned to the first in the time series. The images were then coregistered and spatially normalized into standard stereotactic space (MNI template). All images were spatially smoothed with an $8 \mathrm{~mm}$ full width-half maximum isotropic Gaussian filter.

ERP data acquisition. The electroencephalogram was recorded using a high-density 64-channel geodesic net from Electrical Geodesics and sampled at $250 \mathrm{~Hz}$, with a bandpass of $0.1-100 \mathrm{~Hz}$. The electrooculogram was recorded from over and under the left eye to monitor blinks and vertical eye movements and from the right outer canthus to monitor horizontal eye movements. Impedances were maintained below $50 \mathrm{k} \Omega$. The averaged ERPs were also digitally filtered off-line with a $30 \mathrm{~Hz}$ low-pass filter. All electrodes were referenced to the vertex during recording and rereferenced off-line to the average of the left and right mastoids for analysis. Artifact rejection was conducted off-line using an automatic computer program to reject trials containing blinks and vertical and horizontal eye movements $( \pm 70 \mu \mathrm{V})$. A mean of $88 \%$ of the trials (mean $=44 / 50$ trials per condition, $\mathrm{SD}=7.06)$ for the $\mathrm{TD}$ group, $70 \%$ (35/50 trials, $\mathrm{SD}=$ $12.71)$ for the WS group, and $64 \%(32 / 50$ trials, $S D=11.05)$ for the $\mathrm{DD}$ group were retained for analysis. The WS group had fewer artifact-free trials per condition than the TD group main effect of group $\left(F_{(1,53)}=\right.$ $10.00, p=0.003)$ but did not differ from the DD group $\left(F_{(1,43)}=0.601\right.$, $p=0.443)$. Two additional participants with WS, one TD, and one DD had $<10$ trials for a given condition and were excluded from the analysis.

fMRI data analysis. Fixed-effects models representing two runs for each participant were used at the individual subject level of analysis and random effects models were used for group-level analyses (SPM2). At the individual level, models were created that represented all event-related task conditions (happy, fearful, neutral, scrambled, and fixation). Each stimulus presentation was modeled as a single event. Data were high-pass filtered. Images identified to correspond with $>2 \mathrm{~mm}$ of motion were not included in the statistical analysis. There was no significant difference in the number of images excluded between groups (TD average $=0.15$; $\mathrm{WS}=0.50)$. Images were not excluded if they corresponded with incorrect responses.

Statistical analyses were initiated by performing a condition-by-group $(2 \times 2)$ interaction analysis. This was conducted by using a random effects model comparing the difference in blood oxygenation leveldependent (BOLD) signal during each emotional condition (happy or fearful) versus neutral between experimental groups (WS vs TD). The simple effects within each experimental group were then explored by comparing BOLD signal change between each of the emotional conditions (happy or fearful) versus neutral.

Based on previous studies reporting atypical amygdala function in WS (Meyer-Lindenberg et al., 2005) and previous studies reporting changes 
in amygdala function during the processing of positive social stimuli (Canli et al., 2002; Skuse et al., 2003; Paton et al., 2006; Costafreda et al., 2008), we designated the amygdala as an a priori region of interest (ROI). The delineation of the left and right amygdala ROIs were based on Talairach definitions in standard stereotactic space (http://www.fmri. wfubmc.edu). Unless otherwise specified, we used a $p<0.05$ statistical threshold (corrected for multiple comparisons within the amygdala) for this analysis. Event-related responses were extracted from significant clusters within the amygdala using a moving average for each time course of $200 \mathrm{~ms}$. Percentage signal change between conditions was calculated based on the difference of the peak response extracted from each cluster during either happy or fearful conditions compared with neutral.

ERP data analysis. Numerous studies have identified ERP components sensitive to emotional content, including the components measured here: an N200 linked to arousal and attention (Streit et al., 1999; Sato et al., 2001; Balconi and Lucciari, 2007), an anterior positivity linked to valence (Dolcos and Cabeza, 2002), and a posterior positivity linked to arousal and/or increased subsequent memory for emotional stimuli (Diedrich et al., 1997; Dolcos and Cabeza, 2002; Eimer and Holmes, 2007; Koenig and Mecklinger, 2008). Based on the current data and previous studies, ERP emotion effects were measured using mean amplitudes for the N200 between 200 and $280 \mathrm{~ms}$, the anterior positivity from 500 to $700 \mathrm{~ms}$, and the posterior positivity from 300 to $500 \mathrm{~ms}$. A peak latency measure was also taken for the N200 between 200 and $280 \mathrm{~ms}$. As the distributions of these emotion effects varied over the scalp, the electrodes used in the statistical analysis differed for each ERP component (supplemental Fig. 1, available at www.jneurosci.org as supplemental material). Trials were not excluded if they corresponded with incorrect responses.

Group comparisons of the difference waveforms for happy - neutral and fearful - neutral for the N200, P300-500, and P500-700 were analyzed in repeated-measures ANOVAs using SPSS 13 with three levels of group (WS, TD, and DD) as the between-subjects variable, two levels of hemisphere (left and right), and electrode site (number is variable according to the montage) as repeated measures. The happy versus neutral comparison was conducted over central posterior sites, and thus there was no comparison across hemisphere. Planned comparisons for each group analyzed separately were conducted for each component with two levels of emotion (e.g., happy vs neutral, or fearful vs neutral), two levels of hemisphere for fearful versus neutral, and electrode site (same sites as used in the group analyses). The Greenhouse-Geisser correction was used for main effects and interactions with more than one electrode site. To control for differences between men and women in the effects of emotion on brain activity, preliminary analyses included gender as a separate between-subjects factor in the ANOVA. There were no interactions with emotion and gender for any of the groups; therefore, gender was not included as a factor in the analyses reported below.

\section{Results}

\section{Behavioral measures}

Mean reaction times and accuracy were submitted to a general linear model of repeated measures with subject group (WS, TD, and DD) and experimental condition (happy, fearful, neutral, and scrambled) entered as factors. Overall, WS subjects (mean reaction time: 1222.66$)$ responded slower compared with TD subjects (796.03) $(F=46.61, p<0.001)$ but not DD (1377.66) subjects $(F=1.35, p=0.25)$. Similarly, WS ( $88.33 \%$ accuracy) subjects were less accurate in identifying gender than TD $(97.36 \%$ accuracy) $(F=0.15 .47, p<0.001)$ subjects, but not DD $(88.97 \%)$ subjects $(F=0.03, p=0.87)$. Participants in each group and in each study (fMRI and ERP) were cognizant of all task instructions and performed the task above a threshold for accuracy of $75 \%$. There was no between-group effect in the difference between reaction time (RT) or accuracy during either of the emotional conditions (RT: happy: $t=-0.73 ; p>0.10$; fearful: $t=0.55 ; p>$ 0.10 ; accuracy: happy: $t=-0.56 ; p>0.10$; fearful: $t=1.36 ; p>$

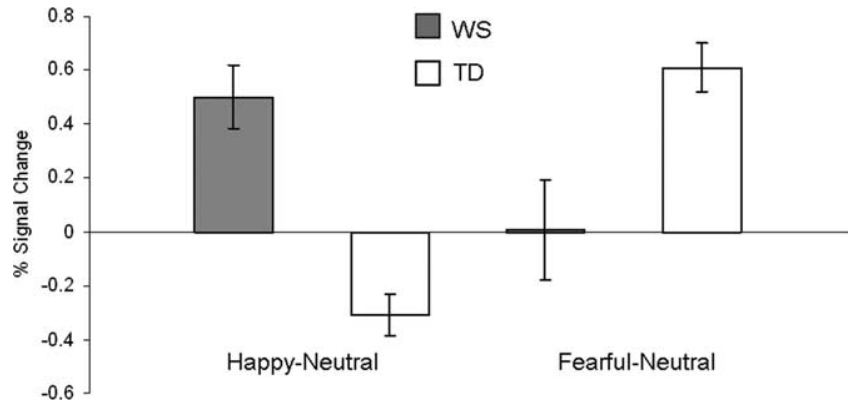

Figure 1. Right amygdala activation to happy (left) and fearful (right) facial expressions compared with neutral between groups. Bars represent percentage signal change of clusters localized by means of direct comparisons between the TD and WS groups. Error bars represent SEM.

0.10 ) compared with the neutral condition (condition $\times$ group interaction).

\section{Amygdala reactivity to happy facial expressions}

We compared happy relative to neutral amygdala response between the two experimental groups (condition $\times$ group interaction). Relative to TD participants, the WS group exhibited greater right amygdala reactivity to happy compared with neutral facial expressions ( $p<0.05$ corrected; 28 voxels; MNI: 30, $0,-14$ ) (Fig. 1, left). On the other hand, the TD group did not exhibit significantly greater left or right amygdala reactivity to happy compared with neutral facial expressions than the WS group. We next compared BOLD signal within the amygdala during the response to happy and neutral facial expressions in the WS and TD groups independently (Fig. 2). The WS group exhibited significant right amygdala reactivity to happy compared with neutral facial expressions ( $p<0.05$ corrected; 16 voxels; MNI: $32,-2$, -14 ) (Fig. 2B). This cluster encompassed a relatively superior region $(z=-14)$ within the standardized amygdala ROI. No significant left or right amygdala activation in response to happy compared with neutral facial expressions was observed in the TD group.

\section{Amygdala reactivity to fearful facial expressions}

We compared fearful relative to neutral amygdala response between the two experimental groups (condition $\times$ group interaction). Relative to WS participants, the TD group exhibited greater right amygdala reactivity to fearful compared with neutral facial expressions at a level approaching statistical significance $(p=$ 0.064 corrected, $p=0.002$ uncorrected, two voxels; MNI: 20, 0 , -14) (Fig. 1, right). To explore the consistency of our findings with that of a previous study (Meyer-Lindenberg et al., 2005), we also compared amygdala response to fearful expressions relative to "nonsocial" stimuli (scrambled faces). Relative to the WS group, the TD group exhibited greater right amygdala reactivity to fearful compared with scrambled faces at the corrected statistical threshold ( $p<0.05$ corrected, 19 voxels; MNI: $24,-2$, $-16)$. There was no significant difference in amygdala reactivity to neutral relative to scrambled faces between the WS and TD groups. Additionally, the WS group did not exhibit significantly greater left or right amygdala reactivity to fearful (compared with either neutral or scrambled) facial expressions than the TD group. We next compared BOLD signal within the amygdala during the response to fearful and neutral facial expressions in the TD and WS groups independently (Fig. 2). The TD group exhibited significant right amygdala reactivity to fearful compared 

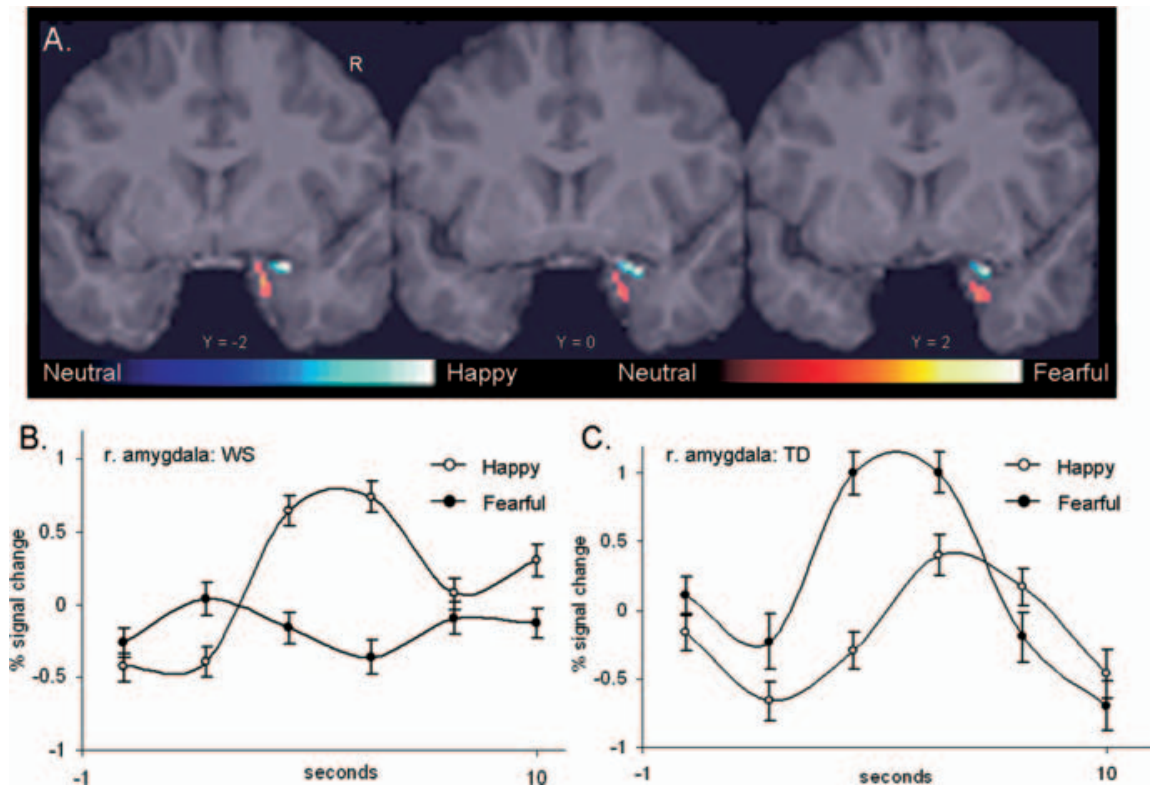

Figure 2. Areas of greater right amygdala reactivity to fearful and happy facial expressions (compared with neutral) within the WS and TD samples. $\boldsymbol{A}$, These clusters were not overlapping. We reduced the threshold to $p<0.05$ uncorrected to visualize the distinct location for each cluster. No voxels within the right amygdala were found to be significantly activated in response to fear versus neutral in the WS group or happy versus neutral in the TD group at the same statistical threshold. Clusters are overlaid onto three coronal slices (Montreal Neurological Institute coordinates: $y=-2,0,2$ ) of a representative TD brain normalized into standard stereotactic space. Voxels of greater activation in response to happy versus neutral facial expressions in the WS group are designated by cool colors (blue). Voxels of greater activation in response to fearful versus neutral facial expressions in the TD group are designated by warm colors (orange). Extracted time course of percentage signal change for peak voxels within the clusters displayed in $\boldsymbol{A}$ are shown in $\boldsymbol{B}$ for WS participants and in $\boldsymbol{C}$ for TD participants. Data were extracted and converted to percentage signal change using a moving average (200s) by a standardized method (xjview toolbox in SPM2). Lines denoted with open circles represent BOLD response to happy faces. Lines denoted with filled circles represent BOLD response to fearful faces. Error bars represent SEM. R, Right.
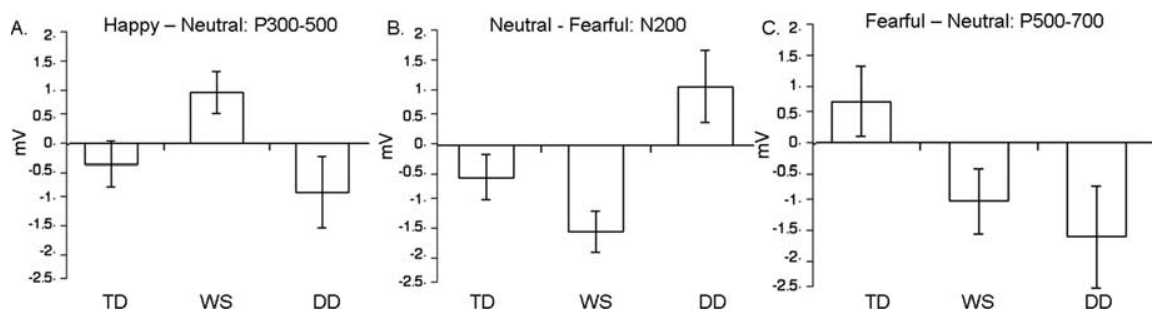

Figure 3. Direct comparison between groups for the $\mathrm{P300}-500$ during happy relative to neutral facial expressions and the N200 and P500-700 during fearful relative to neutral facial expressions. $\boldsymbol{A}$, The P300-500 difference for happy - neutral was larger for WS than TD or DD groups. $\boldsymbol{B}$, For the N200, the WS group showed a larger N200 difference to neutral - fearful than TD or DD groups. C, The P500-700 difference to fearful - neutral was larger for TD than WS or DD groups.

with neutral ( $p<0.05$ corrected; 12 voxels; MNI coordinates: 20 , $-6,-20)$ (Fig. $2 C$ ) and scrambled ( $p<0.05$ corrected; 39 voxels; MNI: 22, $-4,-22)$ facial expressions. On the other hand, no significant left or right amygdala activation to fearful compared with neutral or scrambled facial expressions was observed in the WS group.

\section{Amygdala response associated with IQ, reaction time, and accuracy}

The fMRI study did not include a developmentally delayed control group as was available in the ERP study. Therefore, within the WS group, we examined the association of amygdala response with cognitive and behavioral variables that differentiated this group from TD controls. First, we entered IQ as a covariate with BOLD signal extracted from the right amygdala and found that there were no significant correlations between IQ and amygdala activity either in response to happy ( $p=0.57$ ) or fearful $(p=0.48)$ facial expressions within the WS group. Second, we entered reaction time and accuracy as covariates with BOLD signal extracted from the right amygdala and again found that there were no significant correlations between either reaction time or accuracy and activity either in response to happy or fearful facial expressions within the WS group (all $p$ values $>0.10$ ).

\section{ERPs}

The organization of the ERP results section is designed to mirror the comparisons reported in the fMRI findings above. Thus, comparisons of ERP mean amplitudes linked to happy versus neutral expressions (P300-500) are followed by fearful versus neutral expressions (N200 and P500-700) respectively. In each section, we first report the overall emotion (happy or fearful vs neutral) $\times$ group $(\mathrm{WS}, \mathrm{TD}, \mathrm{DD})$ interaction for a given ERP component. This is followed by tests of the predicted group differences for specific ERP emotion effects by using difference waves (e.g., P500 to happy minus neutral). These a priori hypotheses are further examined for each group individually. Finally, the specificity of the targeted emotion effects is examined for each ERP component. That is, where a significant emotion (happy or fearful vs neutral) $\times$ group $(\mathrm{WS}, \mathrm{TD}, \mathrm{DD})$ interaction is observed, we conduct the same condition $\times$ group interaction analysis but substitute happy with fearful or fearful with happy. Throughout the ERP results section, $p$ values that are $<0.05$ are referred to as significant, $0.05-0.10$ as trends, and $>0.10$ as nonsignificant.

\section{ERPs to happy facial \\ expressions: P300-500}

Based on visual inspection of the ERPs and previous research, ERPs to happy versus neutral expressions were examined from 300 to $500 \mathrm{~ms}$ (P300500 ) for the WS, TD, and DD groups over six posterior sites (EGI locations, left: 29, 33, 42; right: 41, 34, 38) (Fig. 3A). The condition $\times$ group interaction indicated that the $\mathrm{P} 300-500$ response to happy versus neutral facial expressions was significantly different between the three groups $\left(F_{(2,66)}=3.61, p=0.03, \eta_{\rho}^{2}=\right.$ 0.100 ) (Fig. 3A). We next tested the a priori hypothesis that the WS group would exhibit a greater $\mathrm{P} 300-500$ response to happy versus neutral facial expressions than the TD and DD control groups. Between-group comparisons showed that the P300-500 difference to happy minus neutral facial expressions was larger for the WS than for the TD group $\left(F_{(1,53)}=7.189, p=0.01, \eta_{\rho}^{2}=\right.$ $0.119)$ or the DD group $\left(F_{(1,42)}=5.362, p=0.026, \eta_{\rho}^{2}=0.113\right)$ but did not differ between the TD and DD groups $\left(F_{(1,37)}=\right.$ 


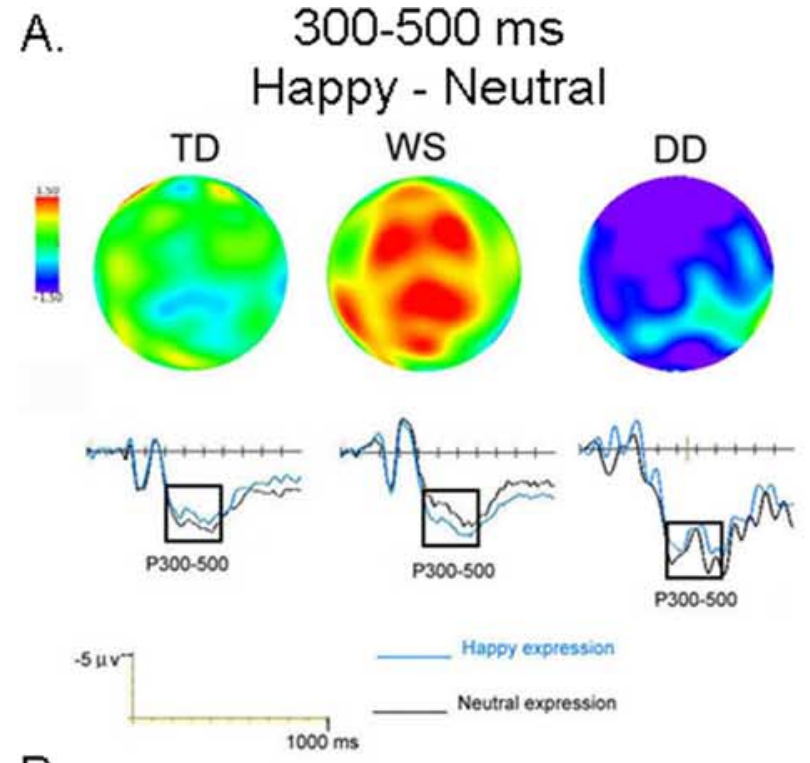

B.
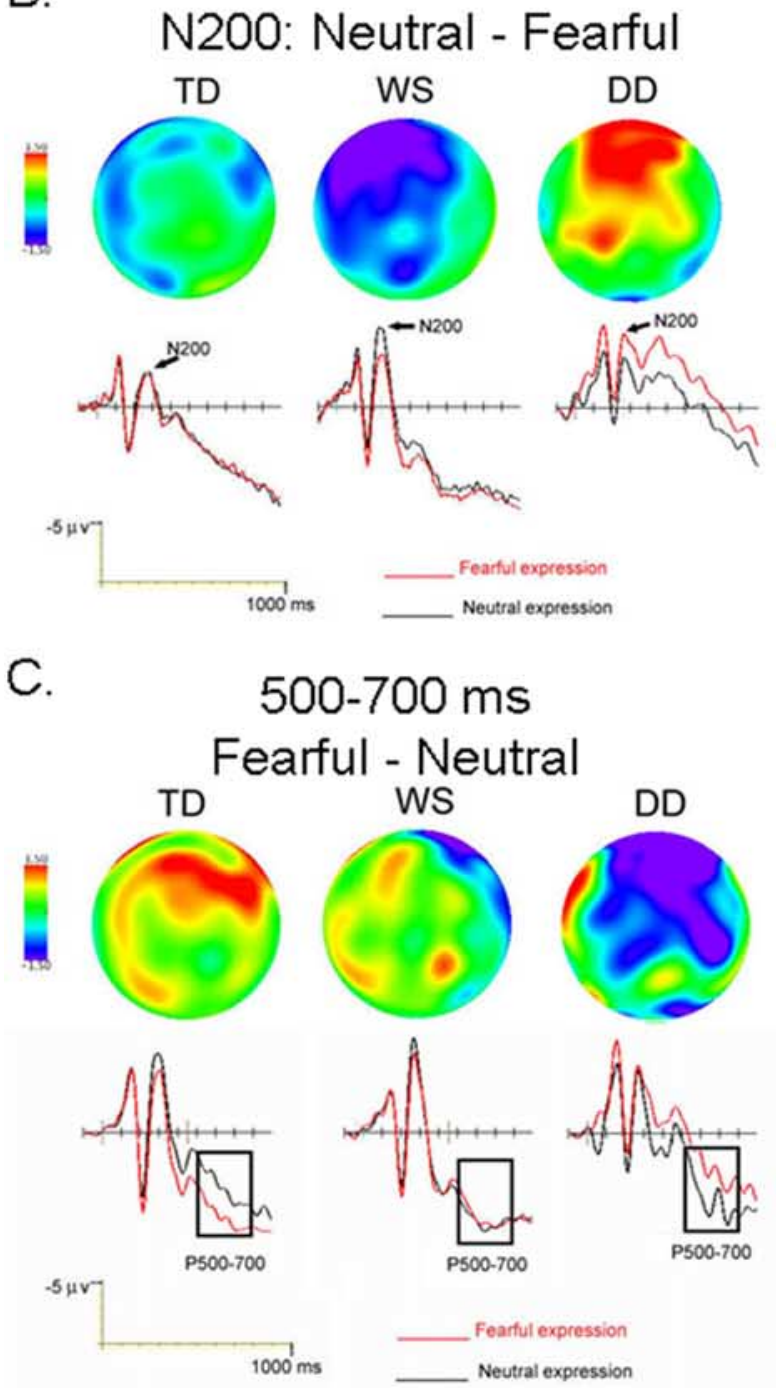

Figure 4. Event-related potentials and topographical maps comparing responses to different emotional expressions for the TD (left), WS (center), and DD (right) groups. Note that negative voltage is plotted up in all ERP plots. Topographical maps (top of each panel) illustrate the distribution of the difference in activity between conditions. Amplitude is plotted as a function of color with the most negative voltage as purple and most positive voltage as red. $A$, ERPs to happy (blue lines) compared with neutral (black lines) expressions depicted at site 42
0.621, $p=0.436)$. We next compared P300 -500 response to happy versus neutral facial expressions in the WS, TD, and DD groups independently (Fig. 4A). The WS group exhibited a greater $\mathrm{P} 300-500$ response to happy versus neutral facial expressions $\left(F_{(1,29)}=4.502, p=0.043, \eta_{\rho}^{2}=0.136\right)$. In contrast, the P300-500 response to happy versus neutral facial expressions was not different for the TD $\left(F_{(1,24)}=0.892, p=0.354\right)$ or DD $\left(F_{(1,14)}=1.817, p=0.201\right)$ groups.

We examined the specificity of emotion by comparing fearful versus neutral P300-500 response over posterior regions between the three (WS, TD, and DD) experimental groups (condition $\times$ group interaction). The condition $\times$ group interaction indicated that the P300-500 response to fearful versus neutral facial expressions was not significantly different between the three groups $\left(F_{(2,66)}=1.426, p=0.248\right)$, nor was the P300-500 comparison to fearful versus neutral expressions significant for any individual group: TD, $F_{(1,24)}=0.046, p=0.832$; WS, $F_{(1,29)}$ $=0.374, p=0.546 ; \mathrm{DD}, F_{(1,14)}=1.435, p=0.252$.

\section{Event-related responses to fearful facial expressions: N200} and P500-700

\section{N200}

We compared the N200 mean amplitude and peak latency to fearful versus neutral expressions for the three groups (WS, TD, and DD) over the left and right hemisphere (total of 16 sites) (see supplemental Fig. 1, available at www.jneurosci.org as supplemental material). The condition $\times$ group interaction indicated that the N200 to fearful versus neutral facial expressions was significantly different between groups in mean amplitude $\left(F_{(2,66)}\right.$ $\left.=4.531, p=0.014, \eta_{\rho}^{2}=0.122\right)$, group $\times$ emotion $\times$ electrode site $\left(F_{(2,66)}=2.363, p=0.034, \eta_{\rho}^{2}=0.067\right)$ (Fig. $\left.3 B\right)$. There were no condition $\times$ group effects for peak latency $\left(F_{(2,66)}=0.290\right.$, $p=0.749$ ). We next tested the a priori hypothesis that the WS group would exhibit decreased neural activity to fearful versus neutral facial expressions relative to the TD and DD control groups. Between-group comparisons showed that the N200 difference wave to fearful minus neutral facial expressions tended to be greater (more negative) for the WS than for the TD group over the left hemisphere $\left(F_{(1,53)}=3.321, p=0.07\right)$ and was greater (more negative) for WS than for the DD group $\left(F_{(1,42)}=8.327\right.$, $p=0.006, \eta_{\rho}^{2}=0.165$ ). In contrast, the effect was smaller (more positive) for the DD than for the TD group $\left(F_{(1,37)}=4822, p=\right.$ $\left.0.034, \eta_{\rho}^{2}=0.115\right)$. We next compared the N200 to fearful versus neutral facial expressions in the WS, TD, and DD groups inde-

\footnotetext{
(P2). For the WS group, the P300 -500 (boxed area) was larger to happy than neutral expressions. The P300-500 did not differ by emotional expression for the other two groups. The posterior central distribution of the happy - neutral difference wave at $350 \mathrm{~ms}$ is shown in the topographical map. $\boldsymbol{B}$, ERPs to neutral (black lines) compared with fearful (red lines) expressions shown at electrode 12 (AF3). The N200 amplitudes to fearful compared with neutral expressions are decreased in the WS group, and increased in the DD group. The topographical maps illustrate the anterior distribution of the N200 difference to fearful - neutral expressions at $240 \mathrm{~ms}$. A larger $\mathrm{N} 200$ to fearful than neutral expression results in a positive difference that is represented in red as in the DD group (right side). In contrast, a smaller N200 to fearful than neutral expression results in a difference that is negative in voltage represented in purple as for the WS group. C, ERPs to neutral (black lines) and fearful (red lines) expressions are shown at the bottom half of the figure shown at electrode 57 (next to $F(4)$ ). The anterior positivity from 500 to $700 \mathrm{~ms}$, P500 -700 (boxed area), was larger to fearful than neutral expression for the TD controls (left) and did not differ for the WS group. For the DD group, the P500-700 appears to be larger to neutral than fearful expressions at this site, but the effect was not significant at this or other sites. The anterior distribution of the P500-700 difference wave to fearful - neutral expression at $520 \mathrm{~ms}$ is illustrated in the topographical maps.
} 
pendently (Fig. $4 B$ ). For the WS group, the N200 was smaller to fearful than neutral expressions over the left hemisphere, condition $\times$ hemisphere $\left(F_{(1,29)}=13.048, p=0.001, \eta_{\rho}^{2}=0.316\right)$, and peaked significantly earlier $\left(F_{(1,29)}=14.350, p=0.001\right)$. In contrast, for the TD group, the N200 did not differ in mean amplitude to fearful versus neutral expressions $\left(F_{(1,24)}=1.253, p=\right.$ $0.214)$ or peak latency $\left(F_{(1,23)}=1.997, p=0.171\right)$. Although the DD group did not show $\mathrm{N} 200$ mean amplitude $\left(F_{(1,14)}=2.089\right.$, $p=0.172)$ or latency $\left(F_{(1,14)}=0.724, p=0.495\right)$ differences over these electrodes, they did show increased N200 amplitudes to fearful relative to neutral expressions over different central (more medial) regions $(13,4,62 ; \mathrm{F} 5, \mathrm{~F} 3, \mathrm{~F} 4)\left(F_{(1,14)}=4.847, p=0.046\right.$, $\left.\eta_{\rho}^{2}=0.272\right)$.

We examined the specificity of the N200 responsiveness to fearful versus neutral emotions by comparing the N200 amplitude to happy versus neutral expressions over the same 16 regions for the three (WS, TD, and DD) experimental groups (condition $\times$ group interaction). The N200 response to happy versus neutral facial expressions was not significantly different between the three groups $\left(F_{(2,66)}=2.185, p=0.121\right)$.

\section{P500-700}

We compared the P500-700 mean amplitude to fearful versus neutral expressions for the three groups at 14 sites (EGI sites, left: $14,12,19,15,13,20,16$; right: 1, 2, 60, 61, 62, 56, 57) (Fig. 3C; supplemental Fig. 1, available at www.jneurosci.org as supplemental material). The predicted emotion $\times$ group $\times$ hemisphere interaction only approached significance $\left(F_{(2,66)}=2.838, p=\right.$ $0.066)$. Based on the current fMRI findings showing increased right amygdala activation to fearful relative to neutral stimuli in the TD group, and previous ERP research showing an increased positivity over right anterior regions in the 500-700 ms range linked to fearful relative to neutral expressions, we predicted that the TD group would show an increased positivity, whereas the WS group would show a diminished positivity, to fearful relative to neutral expressions. Therefore, we tested the a priori hypothesis that the WS group would exhibit decreased response to fearful versus neutral facial expressions relative to the TD and DD control groups. Between-group comparisons showed that the P500-700 difference to fearful minus neutral facial expressions was larger for the TD group than for the WS groups over right anterior regions $\left(F_{(1,53)}=4.337, p=0.042, \eta_{\rho}^{2}=0.076\right)$. The P500-700 difference to fearful minus neutral facial expressions did not significantly differ between the WS and the DD groups $\left(F_{(1,42)}=1.717, p=0.197\right)$ at any electrode site. However, there was a trend for the P500-700 difference to fearful minus neutral facial expressions to be different between the TD and DD groups $\left(F_{(1,37)}=3.329, p=0.08\right)$. We next compared P500-700 response to fearful versus neutral facial expressions in the WS, TD, and DD groups independently (Fig. 4C). For the WS group, the P500-700 response to fearful versus neutral facial expressions was smaller over the right hemisphere $\left(F_{(1,29)}=6.376, p=0.017\right.$, $\left.\eta_{\rho}^{2}=0.180\right)$. For the TD group, the P500-700 response to fearful versus neutral facial expressions tended to be larger over right anterior regions $\left[F_{(1,24)}=3.335, p=0.08, \eta_{\rho}^{2}=0.115\right.$; at electrode $\left.57(\mathrm{FC} 6), t_{(24)}=2.342, p=0.028\right]$. For the DD group, the P500-700 response to fearful versus neutral facial expressions was not significantly different at any site $\left(F_{(1,14)}=1.345, p=\right.$ $0.267)$.

We examined the specificity of emotion by comparing happy versus neutral P500-700 response over the same regions between the three (WS, TD, and DD) experimental groups. The emotion $\times$ group interactions indicated that the P500-700 response to happy versus neutral facial expressions was not significantly different between the three groups $\left(F_{(2,66)}=1.081, p=0.345\right.$; group $\times$ emotion $\times$ hemisphere, $\left.F_{(2,66)}=0.152, p=0.860\right)$.

\section{Discussion}

Results from the fMRI and ERP studies using identical stimuli and a similar experimental design provided convergent evidence that individuals with WS exhibit both heightened amygdala reactivity to happy facial expressions and diminished amygdala reactivity to fearful facial expressions. The heightened amygdala response to socially appetitive stimuli may be a fundamental neural substrate that underlies atypical social drive in WS. Similar to the results of a previous study (Meyer-Lindenberg et al., 2005), we also found that TD healthy controls exhibited a robust amygdala response to fearful facial expressions, while the WS group did not, although the condition $\times$ group interaction (using neutral as a baseline) approached corrected statistical significance. By using an additional methodological approach (ERPs), we were able to provide an electrophysiological correlate of these fMRI findings and to examine the temporal specificity of the neural response to fearful stimuli within each group. Specifically, the WS group showed decreased activity to fearful versus neutral expressions as reflected by decreases in the mean amplitudes of the N200, compared with both the DD and TD groups. The significant betweengroup differences in N200 amplitude provide evidence that relatively early during the visual and perceptual stream, WS individuals differ from both healthy and IQ-matched controls in how fearful facial expressions are processed.

Of particular interest here was that the WS group showed increased activity to happy relative to neutral expressions as evidenced by relative increases in BOLD signals in the right amygdala and amplitude of the posterior P300-500 waveform. The posterior P300-500 ERP effect has been linked to enhanced memory for emotional content and may reflect increased attention to emotionally salient stimuli during encoding (Deidrich et al., 1997; Dolcos and Cabeza, 2002; Koenig and Mecklinger, 2008). Regarding the ERPs to fearful faces, we did not predict that the P500-700 difference to fearful minus neutral facial expressions would be different between the TD and DD groups. An ERP/fMRI study of positive, negative, and emotional scenes showed that the amplitude of the late posterior positivity indexes emotional intensity (Sabatinelli et al., 2007) and is correlated with activation from the amygdala (Sabatinelli et al., 2005). The present data represent a new finding and are the first to show increased neural activity to positive emotional expressions in WS.

Considering the fMRI findings for both positively and negatively valenced social stimuli, the clusters of fMRI activation within the right amygdala were observed to be nonoverlapping (Fig. 2A). This suggests that distinct neuronal populations may subserve these functions and that this effect may be primarily lateralized to the right amygdala. Recently, single-unit recording studies in primates have indicated the presence of emotion selective neurons within the amygdala (Paton et al., 2006; Kuraoka and Nakamura, 2007). Although some lesion studies in rats indicate that disparate nuclei within the amygdala are engaged in different functions (Knapska et al., 2007; Yang et al., 2008), other studies suggest that amygdala nuclei function in parallel during appetitive and aversive processing (Balleine and Killcross, 2006). The fact that the results in this study are based on averaged, spatially smoothed data limits how precisely the clusters can be assigned to specific nuclei within the amygdala. However, a single-subject analysis based on nonsmoothed data confirms the general topographical dissociation of amygdala activation be- 
tween groups but also demonstrates the heterogeneity of BOLD signal within groups (supplemental Fig. 2, available at www. jneurosci.org as supplemental material). The putative dissociation of these loci suggests that the genetic deletion in WS may influence the development and function of separate amygdala regions (and possibly nuclei) differently.

The increased amygdala response to happy facial expressions in individuals with WS may represent several psychological processes linked to the amygdala including attention, arousal, or anxiety. For example, the central nucleus of the amygdala has been strongly linked with attention (Holland and Gallagher, 1999; Maddux et al., 2007). Although eye-tracking information would have helped clarify this issue in our study, unfortunately, equipment to obtain these data were not available at the time of subject scanning. However, other investigations have assessed attentional mechanisms during social processing in WS by measuring gaze duration of infants and toddlers toward faces (Mervis et al., 2003), or by performing eye tracking when subjects viewed social and nonsocial stimuli (Riby and Hancock, 2008). The results of these studies indicate that WS is associated with enhanced attention for social and emotional stimuli. For example, whereas individuals with autism tend to exhibit reduced fixation toward faces and eyes, people with WS exhibit heightened amounts of fixation toward faces and eyes (Riby and Hancock, 2008) relative to typically developing and nonverbal ability-matched controls. Although demonstrating a WS-associated predisposition for increased attention to social-emotional stimuli in general, these studies did not directly address differential attention for or neural responses to positive versus negative emotional stimuli in affected individuals.

The social phenotype in WS has been described as being comprised of relative strengths and weaknesses (Mervis, 2003; Feinstein and Singh, 2007). On one hand, those with WS appear to be particularly driven toward engaging in social interaction and to approach others such as strangers excessively and inappropriately. Neuropsychological research has demonstrated that individuals with WS tend to rate happy facial expressions as more approachable relative to other emotions than do mentally aged controls (Frigerio et al., 2006; Porter et al., 2007). On the other hand, those with WS often experience profound difficulties maintaining relationships and also experience heightened levels of anxiety during social interaction (Dykens, 2003). The data from the current study is consistent with this behavioral phenotype of WS and provides neurobiological evidence that the WS social phenotype is mediated by both increased neural reactivity to happy expressions and decreased reactivity to fearful expressions. The heightened amygdala activity during the processing of happy facial expressions in WS may be a neural marker of anticipatory processes that precede events during which the likelihood of social engagement is high.

Our current study is limited by several constraints defined by our subject groups and experimental design. WS is often associated with general delays in cognitive development. In our ERP study, we took advantage of an IQ-matched (developmentally delayed) control group to demonstrate that the observed responsiveness to social stimuli was not driven by IQ. Due to limited recruitment that yielded poor statistical power, we were not able to use such a control group for our fMRI study. However, we undertook several procedures to investigate whether the observed differences between the WS and TD group in the PMRI study were driven by differences in either IQ or behavioral responses. The findings from these analyses, as well as from the ERP study that included DD controls, indicate that differences in IQ or task performance are not driving the significant betweengroup fMRI findings in amygdala response to positive and negative social stimuli.

Our data provide evidence that a fundamental characteristic of the WS phenotype consists of aberrant neural reactivity to emotional facial expressions. It is unclear, however, what specific components of facial expressions may correspond with this differential response. Previous studies have reported impaired (Gagliardi et al., 2003; Plesa-Skwerer et al., 2006), as well as preserved (Santos et al., 2008), functioning in face recognition in WS. Additionally, in terms of social cognition, it has been speculated that those with WS exhibit a relatively preserved ability to empathize with others (Mervis and Klein-Tasman, 2000) and that they tend to be relatively socially anxious (Dykens, 2003). Future developments in neuropsychological assessment techniques may strengthen our understanding of the relationship between these psychological constructs and the differences in neural activation reported here.

Advancements in social cognitive neuroscience have demonstrated that brain responses to emotional stimuli vary according to task instructions and states, such as mood (Haas and Canli, 2008). In a previous study, Meyer-Lindenberg et al. (2005) provided evidence of atypical amygdala function during social processing by using a matching task (faces vs shapes). Our study used similar negative (fearful) social stimuli but also included positive (happy) social stimuli and a different set of task instructions (gender discrimination). The convergence between the patterns of results between the two types of tasks strengthens the hypothesis that amygdala abnormality is a primary neural substrate that underlies the social phenotype in WS. Continued research that uses different task instructions and assesses other betweensubject characteristics, such as mood and personality, will further advance the existing model of WS and social cognitive brain function.

In summary, this article provides new fMRI and ERP evidence that persons with WS exhibit heightened amygdala and cortical response to happy facial expressions and extends previous research that demonstrated absent or reduced response to fear in WS. This finding provides a neural correlate to previous behavioral findings reporting the tendency to be overly socially appetitive in WS. Future studies will extend our understanding of the genetic influences of social behavior in WS and the interaction between the amygdala and other brain regions associated with social cognition.

\section{References}

Aggleton J P (Ed.) (2000) The amygdala: a functional analysis, Ed 2. Oxford: Oxford UP.

Balconi M, LucciariS (2007) Consciousness and emotional facial expression recognition: subliminal/supraliminal stimulation effect on N200 and P300 ERPs. J Psychophysiol 21:100-108

Balleine BW, Killcross S (2006) Parallel incentive processing: an integrated view of amygdala function. Trends Neurosci 29:272-279.

Bellugi U, Adolphs R, Cassady C, Chiles M (1999) Towards the neural basis for hypersociability in a genetic syndrome. Neuroreport 10:1653-1657.

Canli T, Sivers H, Whitfield SL, Gotlib IH, Gabrieli JD (2002) Amygdala response to happy faces as a function of extraversion. Science $296: 2191$

Costafreda SG, Brammer MJ, David AS, Fu CH (2008) Predictors of amygdala activation during the processing of emotional stimuli: a metaanalysis of 385 PET and fMRI studies. Brain Res Rev 58:57-70.

Derogatis LR (1977) SCL-90: administration, scoring and procedures manual for the revised version and other instruments of the psychopathology rating scale series. Baltimore: John Hopkins University.

Deutsch SI, Rosse RB, Schwartz BL (2007) Williams syndrome: a genetic deletion disorder presenting clues to the biology of sociability and clinical challenges of hypersociability. CNS Spectr 12:903-907. 
Diedrich O, Naumann E, Maier S, Becker G (1997) A frontal positive slow wave in the ERP associated with emotional slides. J Psychophysiol $11: 71-84$.

Dolcos F, Cabeza R (2002) Event-related potentials of emotional memory: encoding pleasant, unpleasant, and neutral pictures. Cogn Affect Behav Neurosci 2:252-263.

Doyle TF, Bellugi U, Korenberg JR, Graham J (2004) "Everybody in the world is my friend" hypersociability in young children with Williams syndrome. Am J Med Genet A 124A:263-273.

Dykens EM (2003) Anxiety, fears, and phobias in persons with Williams syndrome. Dev Neuropsychol 23(1-2):291-316.

Eimer M, Holmes A (2007) Event-related brain potential correlates of emotional face processing. Neuropsychologia 45:15-31.

Feinstein C, Singh S (2007) Social phenotypes in neurogenetic syndromes. Child Adolesc Psychiatr Clin N Am 16:631-647.

Frigerio E, Burt DM, Gagliardi C, Cioffi G, Martelli S, Perrett DI, Borgatti R (2006) Is everybody always my friend? Perception of approachability in Williams syndrome. Neuropsychologia 44:254-259.

Gagliardi C, Frigerio E, Burt DM, Cazzaniga I, Perrett DI, Borgatti R (2003) Facial expression recognition in Williams syndrome. Neuropsychologia 41:733-738.

Haas BW, Canli T (2008) Emotional memory function, personality structure and psychopathology: a neural system approach to the identification of vulnerability markers. Brain Res Rev 58:71-84.

Holland PC, Gallagher M (1999) Amygdala circuitry in attentional and representational processes. Trends Cogn Sci 3:65-73.

Jones W, Bellugi U, Lai Z, Chiles M, Reilly J, Lincoln A, Adolphs R (2000) II. Hypersociability in Williams syndrome. J Cogn Neurosci 12 [Suppl 1]:30-46.

Klein-Tasman BP, Mervis CB (2003) Distinctive personality characteristics of 8-, 9-, and 10-year-olds with Williams syndrome. Dev Neuropsychol 23:269-290.

Koenig S, Mecklinger A (2008) Electrophysiological correlates of encoding and retrieving emotional events. Emotion 8:162-173.

Kuraoka K, Nakamura K (2007) Responses of single neurons in monkey amygdala to facial and vocal emotions. J Neurophysiol 97:1379-1387.

Levitin DJ, Menon V, Schmitt JE, Eliez S, White CD, Glover GH, Kadis J, Korenberg JR, Bellugi U, Reiss AL (2003) Neural correlates of auditory perception in Williams syndrome: an fMRI study. Neuroimage 18:74-82.

Maddux JM, Kerfoot EC, Chatterjee S, Holland PC (2007) Dissociation of attention in learning and action: effects of lesions of the amygdala central nucleus, medial prefrontal cortex, and posterior parietal cortex. Behav Neurosci 121:63-79.

Mervis CB (2003) Williams syndrome: 15 years of psychological research. Dev Neuropsychol 23(1-2):1-12.

Mervis CB, Becerra AM (2007) Language and communicative development in Williams syndrome. Ment Retard Dev Disabil Res Rev 13:3-15.

Mervis CB, Klein-Tasman BP (2000) Williams syndrome: cognition, per- sonality, and adaptive behavior. Ment Retard Dev Disabil Res Rev 6:148-158

Mervis CB, Morris CA, Klein-Tasman BP, Bertrand J, Kwitny S, Appelbaum LG, Rice CE (2003) Attentional characteristics of infants and toddlers with Williams syndrome during triadic interactions. Dev Neuropsychol 23:243-268.

Meyer-Lindenberg A, Kohn P, Mervis CB, Kippenhan JS, Olsen RK, Morris CA, Berman KF (2004) Neural basis of genetically determined visuospatial construction deficit in Williams syndrome. Neuron 43:623-631.

Meyer-Lindenberg A, Hariri AR, Munoz KE, Mervis CB, Mattay VS, Morris CA, Berman KF (2005) Neural correlates of genetically abnormal social cognition in Williams syndrome. Nat Neurosci 8:991-993.

Mobbs D, Garrett AS, Menon V, Rose FE, Bellugi U, Reiss AL (2004) Anomalous brain activation during face and gaze processing in Williams syndrome. Neurology 62:2070-2076.

Paton JJ, Belova MA, Morrison SE, Salzman CD (2006) The primate amygdala represents the positive and negative value of visual stimuli during learning. Nature 439:865-870.

Plesa-Skwerer D, Faja S, Schofield C, Verbalis A, Tager-Flusberg H (2006) Perceiving facial and vocal expressions of emotion in individuals with Williams syndrome. Am J Ment Retard 111:15-26.

Porter MA, Coltheart M, Langdon R (2007) The neuropsychological basis of hypersociability in Williams and Down syndrome. Neuropsychologia 45:2839-2849.

Riby DM, Hancock PJ (2008) Viewing it differently: social scene perception in Williams syndrome and autism. Neuropsychologia 46:2855-2860.

Sabatinelli D, Bradley MM, Fitzsimmons JR, Lang PJ (2005) Parallel amygdala and inferotemporal activation reflect emotional intensity and fear relevance. Neuroimage 24:1265-1270.

Sabatinelli D, Lang PJ, Keil A, Bradley MM (2007) Emotional perception: correlation of functional MRI and event-related potentials. Cereb Cortex 17:1085-1091.

Santos A, Rondan C, Milne D, Demonet JF, Deruelle C (2008) Social relevance boosts context processing in Williams syndrome. Dev Neuropsychol 33:553-564.

Sato W, Kochiyama T, Yoshikawa S, Matsumura M (2001) Emotional expression boosts early visual processing of the face: ERP recording and its decomposition by independent component analysis. Neuroreport 12:709-714.

Skuse D, Morris J, Lawrence K (2003) The amygdala and development of the social brain. Ann N Y Acad Sci 1008:91-101.

Streit M, Ioannides AA, Liu L, Wölwer W, Dammers J, Gross J, Gaebel W, Müller-Gärtner HW (1999) Neurophysiological correlates of the recognition of facial expressions of emotion as revealed by magnetoencephalography. Brain Res Cogn Brain Res 7:481-491.

Zitzer-Comfort C, Doyle T, Masataka N, Korenberg J, Bellugi U (2007) Nature and nurture: Williams syndrome across cultures. Dev Sci 10:755762 